\title{
Association of Biofilm Formation with Antimicrobial Resistance Among the Acinetobacter Species in A Tertiary Care Hospital in Bangladesh
}

\author{
AZIZUN NAHAR, SHAHEDA ANWAR, MD. RUHUL AMIN MIAH
}

\begin{abstract}
Purpose: The purpose of this study was to detect biofilm formation in clinical isolates of Acinetobacter species and to observe correlation between biofilm formation and antimicrobial resistance among Acinetobacter isolates.

Methods: Two hundred fifty six clinical samples collected from patients who were admitted in Intensive Care Unit (ICU) and on device, patients from Surgery, Medicine, Gynae \& Obs and Urology department of Bangabandhu Sheikh Mujib Medical University (BSMMU) and from Burn unit of Dhaka Medical College Hospital were included in this study. Biofilm formation and antibiotyping were performed for the isolates of Acinetobacter species recovered from clinical samples including tracheal aspirates, blood, urine, wound swab, pus, throat swab, endotracheal tubes, burn samples, ascitic fluid, sputum, aural swab, oral swab, cerebrospinal fluid, and catheter tip. Correlation of biofilm formation with antimicrobial resistance pattern among Acinetobacter isolates were also observed in this study.

Result: A total of 256 various specimens were studied of which 95 Intensive Care Unit (ICU) and I6I Non ICU samples. Out of 95 ICU and I6I Non ICU samples, Acinetobacter species were isolated from 32 (33.7\%) and 20(I 2.4\%) respectively. From 32 ICU and 20 Non ICU Acinetobacter isolates, 28 (87.5\%) and II (55\%) were biofilm producers. Biofilm forming capacity of Acinetobacter species was significantly $(p<0.008)$ greater in ICU than in Non ICU isolates. In both ICU and Non ICU isolates, biofilm forming Acinetobacter species were 100\% resistant to amoxicillin, ceftriaxone, ceftazidime, cefotaxime, cefuroxime, and aztreonam. Resistance to antibiotics such as gentamicin, amikacin, netilmicin, ciprofloxacin and imipenem was higher among biofilm forming Acinetobacter isolates in ICU than Non ICU isolates. Susceptibility to colistin was 100\% in Non ICU isolates but in ICU it showed $7.1 \%$ resistance.

Conclusions: This investigation showed that most of the clinical isolates of Acinetobacter species were biofilm producers especially from ICU samples and they were multidrug resistant. Even polymixin resistant Acinetobacter isolates are slowly emerging. This is very alerming for us that biofilm forming multidrug resistant Acinetobacter species represents a severe threat in the treatment of hospitalized patients. So, antibiotic policy and guidelines are essential to eliminate major outbreak in future.
\end{abstract}

Keywords: Acinetobacter, biofilm, multidrug resistance.

\section{Introduction}

Acinetobacter species has emerged as an important nosocomial pathogen as it is the causative agent of several types of infections including pneumonia, meningitis, septicaemia, and urinary tract infections and also responsible for causing intermittent outbreaks especially in ICU. They ranked second after Pseudomonas aeruginosa among the nosocomial, aerobic, non- fermentative, gram negative bacilli pathogens. ${ }^{1,2}$ Infections caused by Acinetobacter are associated with medical devices, e.g. vascular catheter, CSF shunt, Foley catheter, surgical interventions etc. ${ }^{3-5}$ The presence and duration of invasive procedures, as well as

1. Department of Microbiology and Immunology, Bangabandhu Sheikh Mujib Medical University, Dhaka, Bangladesh.

Correspondence : Dr. Azizun Nahar, Senior Lecturer, Department of Microbiology, Bangladesh Medical College, Dhaka, Bangladesh, Email:drnahar.a_26@yahoo.com exposure to broad spectrum antibiotics, have been identified as risk factors for acquisition of Acinetobacter in numerous studies. ${ }^{6}$ Infections caused by them are difficult to control due to multidrug resistance, which limit therapeutic options in critically ill and debilitated patients especially from the intensive care unit, where its prevalence is most noted. ${ }^{2}$

Acinetobacter infections are associated closely with surgery or the use of artificial devices. Patients become infected following initial colonization. This process is influenced by various risk factors, particularly in ICUs, where multiple manipulations following surgery, as well as the use of endotracheal tubes and intravascular, ventricular or urinary catheters, can result in colonization by opportunistic bacteria such as Acinetobacter. 6

Acquisition of the ability to form biofilm could be a good strategy to enhance a microorganism's survival under 
stressed conditions, e.g., during host invasion or following antibiotic treatment. This is because cells growing in biofilms are highly resistant to numerous types of antimicrobial agent. In addition, the ability of horizontal gene transfer of bacterial cells is enhanced within biofilm communities, thereby facilitating the spread of antibiotic resistance. The high colonizing capacity of $A$. baumannii, combined with its resistance to multiple drugs, contribute to the organism's survival and further dissemination in the hospital setting. ${ }^{7}$ The present study was undertaken on clinical isolates of Acinetobacter species to determine biofilm formation and to observe correlation between biofilm formation and antimicrobial resistance among Acinetobacter species.

\section{Material and methods}

Clinical samples were collected from patients admitted in Bangabandhu Sheikh Mujib Medical University (BSMMU) and from patients admitted in Burn unit of Dhaka Medical college. Two hundred fifty six clinical samples included tracheal aspirates, blood from central venous catheter (CVC), peripheral blood, urine, wound swab, pus, throat swab, endotracheal tubes, burn samples, ascitic fluid, sputum, aural swab, oral swab, cerebrospinal fluid, and catheter tip were collected. Out of 256 samples 95 Intensive Care Unit (ICU) samples were collected from patient admitted in ICU and on device and 161 Non ICU samples were collected from Medicine, Surgery, Gynaecology \& Obstetrics, Urology department of BSMMU and from patients admitted in the Burn unit of Dhaka Medical College Hospital. Laboratory works were performed in the department of Microbiology \& Immunology, BSMMU, Dhaka between January 2010 to December 2010.

\section{Isolation and identification of Acinetobacter}

Typical colonies were enumerated, selected and examined further. Acinetobacter was identified by Gram staining, motility, oxidase, catalase, citrate utilization, indole and urease tests, glucose oxidation in Krigler Iron Agar (KIA) media and biochemical tests in oxidation and fermentation media (OF media). These identification schemes were done as per standard techniques. ${ }^{8,9}$

\section{Antimicrobial susceptibility tests}

All the Acinetobacter isolates from BSMMU \& Dhaka medical college hospital were tested for antimicrobial susceptibility testing by disc diffusion method using the Kirby-Bauer technique ${ }^{10}$ and as per recommendations of the National Committee for Clinical laboratory Standards (NCCLS). ${ }^{11}$ Antimicrobial disks used for sensitivity tests were amoxicillin(10mg), ciprofloxacin(5mg), gentamicin (10mg), ceftriaxone (30mg), ceftazidime $(30 \mathrm{mg})$, cefuroxime (30mg), cefotaxime (30mg), amikacin $(30 \mathrm{mg})$, aztreonam(30mg), imipenem(10mg), netilmicin(30mg) and colistin( $10 \mathrm{mg})$ were used respectively.

\section{Detection of biofilm}

The ability of Acinetobacter isolates to form biofilm was performed as described by Toledo et al., 2001. ${ }^{12}$ Isolates were grown over night at $37^{\circ} \mathrm{C}$ in Brain Heart Infusion Broth (BHIB) with $0.25 \%$ glucose. The culture was diluted at a ratio of 1:20 in fresh Brain Heart Infusion broth (BHIB) with $0.25 \%$ glucose. $200 \mu 1$ of this suspension was used to inoculate in sterile 96 well flat bottomed polystyrene microtiter plate. Then the plate was incubated at $37^{\circ} \mathrm{C}$ for 24 hours. Wells were washed with Phosphate buffer solution (PBS) three times. Non-adherent cells were removed by washing with phosphate buffer. Then the microtiter plate was dried in an inverted position. After that plate was stained with $0.5 \%$ Crystal violet (CV) for 15 minutes. Wells were rinsed once more. Then $200 \mu$ l ethanol/acetone (80:20, v/v) were added in each well to solubilize CV. The optical density (OD) was determined using a microtiter reader. Each assay was performed in triplicate $\&$ repeated twice. The average OD values were calculated for all tested strains and negative controls, since all tests were performed in triplicate and repeated three times. Second, the cut off value (ODc) was established. It was defined as three standard deviations (SD) above the mean OD of the negative control: ODc=average OD of negative controls + (3XSD of negative control). Final OD value of a tested strain was expressed as average OD value of the strain reduced by ODc value $(\mathrm{OD}=$ average $O D$ of a strain - ODc). ODc value was calculated for each microtiter plate separately. Any negative value was presented as zero, while any positive value was indicated biofilm production. ${ }^{13}$

\section{Results}

A total of 256 various specimens were studied which included 95 ICU and 161 Non ICU samples. Out of 95 ICU and 161 Non ICU samples, Acinetobacter species were isolated from 32 (33.7\%) and 20(12.4\%) respectively. In ICU, Acinetobacter species were predominantly isolated from endotracheal tube (100.0\%) followed by tracheal aspirate $(54.3 \%)$, blood from central venous catheter blood $(36.4 \%)$, peripheral blood (13.6\%) and urine (12.5\%). In Non ICU, Acinetobacter species were isolated from wound swab $(25.0 \%)$, pus $(13.9 \%)$, peripheral blood $(50 \%)$, urine $(44.4 \%)$ and throat swab (11.1\%). No growth of Acinetobacter species were detected in other samples namely ascitic fluid, sputum, aural swab, oral swab, burn samples, cerebrospinal fluid and catheter tips. From 32 ICU and 20 Non ICU Acinetobacter isolates 28 $(87.5 \%)$ and $11(55.0 \%)$ were biofilm producers. Biofilm forming capacity of Acinetobacter species was significantly $(\mathrm{p}<0.008)$ higher in ICU than Non ICU isolates. The rate of biofilm production by isolated Acinetobacter species from different clinical samples is shown in Table-1.

From ICU and Non ICU samples all the biofilm forming Acinetobacter isolates were $100 \%$ resistant to amoxicillin, ceftriaxone ceftazidime, cefotaxime, cefuroxime and 
aztreonam. Higher level of resistance was also recorded in Table-2. Only colistin showed $7.1 \%$ resistance in biofilm forming Acinetobacter isolates in ICU and 100\% sensitivity in Non ICU isolates.

Table-I

Biofilm production of isolated Acinetobacter species from different clinical samples

\begin{tabular}{lccc}
\hline ICU samples & $\begin{array}{c}\text { Total } \\
\text { No. samples }\end{array}$ & $\begin{array}{c}\text { Positive for } \\
\text { Acinetobacter } \\
\text { species } \\
\mathrm{N}(\%)\end{array}$ & $\begin{array}{c}\text { Positive for } \\
\text { production } \\
\text { biofilm }\end{array}$ \\
N (\%) \\
\hline Tracheal aspirate & 35 & $19(54.3)$ & $16(84.2)$ \\
Blood CVC & 11 & $4(36.4)$ & $4(100.0)$ \\
Peripheral blood & 22 & $3(13.6)$ & $3(100.0)$ \\
Urine & 24 & $3(12.5)$ & $3(100.0)$ \\
Endotracheal tube & 3 & $3(100.0)$ & $2(66.7)$ \\
Total & 95 & $32(33.7)$ & $28(87.5)$ \\
Non ICU samples & & & \\
Wound swab & 32 & $8(25.0)$ & $4(50.0)$ \\
Pus & 36 & $5(13.9)$ & $2(40.0)$ \\
Peripheral blood & 4 & $2(50.0)$ & $2(100.0)$ \\
Urine & 9 & $4(44.4)$ & $3(75.0)$ \\
Throat swab & 9 & $1(11.1)$ & $0(0.0)$ \\
Others & 71 & $0(0.0)$ & $0(0.0)$ \\
\hline Total & 161 & $20(12.4)$ & $11(55.0)$ \\
\hline
\end{tabular}

Table-II

The antibiotic resistance patterns of biofilm producing Acinetobacter isolates

\begin{tabular}{lcc}
\hline Antibiotics & $\begin{array}{c}\text { Biofilm forming } \\
\text { showing antibiotic resistance (\%) } \\
\text { ICU }\end{array}$ & Non ICU \\
\hline Amoxycillin & 100.0 & 100.0 \\
Ceftriaxone & 100.0 & 100.0 \\
Ceftazidime & 100.0 & 100.0 \\
Cefotaxime & 100.0 & 100.0 \\
Cefuroxime & 100.0 & 100.0 \\
Gentamicin & 100.0 & 88.9 \\
Amikacin & 85.7 & 55.6 \\
Netilmicin & 85.7 & 11.1 \\
Ciprofloxacin & 82.1 & 54.4 \\
Imipenem & 81.0 & 22.2 \\
Aztreonam & 100.0 & 100.0 \\
Colistin & 7.1 & 0.0 \\
\hline
\end{tabular}

\section{Discussion}

Acinetobacter infections present a global medical challenge. They are opportunistic pathogens and are particularly successful at colonizing and persisting in the hospital environment. $^{3-5}$ Biofilm formation is thought to be a key pathogenic feature, especially in relation to intravascular line infections and ventilated associated pneumonia. Generally, two properties are often associated with biofilm producing bacteria, namely, the increased synthesis of exopolysaccharide (EPS) and the development of antibiotic resistance. ${ }^{14}$ One can assume that increased production of EPS in Acinetobacter is likely to create a protective environment leading to difficulty in antibiotic penetration leading to development of resistance. In addition, there appears to be some differences in the cellular physiology of cells within the biofilm that also results in increased drug resistance. ${ }^{15}$ Thus infections due to bacteria that form biofilm is a tenacious clinical problem. In this work, biofilm formation by Acinetobacter isolates were tested and tried to correlate them with antimicrobial resistance.

In this current study, the high isolation rates of Acinetobacter species of about $100 \%$ from endotracheal tube, followed by $54.3 \%$ from tracheal isolates, $36.4 \%$ from central venous catheter blood in ICU and 50\% from peripheral blood, 44.4\% from urine and $25 \%$ from wound swab and $13.9 \%$ from pus in non ICU samples. In India, a study reported that, the high isolation rate of Acinetobacter species of about $24 \%$ were from tracheal aspirates, $16 \%$ from sputum, $12 \%$ from endotracheal tube, $12 \%$ from wound swab and $6 \%$ from blood. ${ }^{16}$

Our study showed $28(87.5 \%)$ and $11(55.0 \%)$ isolates were biofilm producers from $32 \mathrm{ICU}$ and 20 Non ICU Acinetobacter species. Higher rate of biofilm production was found in patients on device in ICU. Present results showed that biofilm plays a role in the pathogenesis of some device-associated Acinetobacter infections. Other study showed that, more than $60 \%$ of $A$. baumannii isolates from clinical samples formed biofilm, and these isolates were associated mainly with device-associated infections. ${ }^{17}$

In this study, Acinetobacter species showed higher biofilm production in central venous catheter blood $100 \%$, peripheral blood $100 \%$, urine $100 \%$ and tracheal aspirates $84.2 \%$ but endotracheal tube showed $66.7 \%$ biofilm production in ICU. In Non ICU isolates, Acinetobacter showed 100\% biofilm formation in peripheral blood, $75 \%$ in urine, $50 \%$ in wound swab and $40 \%$ in pus. Another study found that, biofilm formation by $A$. baumannii were $76.47 \%$ in tracheal aspirate, $80.0 \%$ in wound swab, $75.0 \%$ in blood, $50.0 \%$ in sputum, $50.0 \%$ in pleural fluid, $75.0 \%$ in urine, $80.0 \%$ in cerebrospinal fluid. ${ }^{18}$ 
This study shows association of biofilm formation with antibiogram of Acinetobacter isolates in ICU and in Non ICU. From both ICU and Non ICU samples, all the biofilm forming Acinetobacter isolates from different clinical sources were $100 \%$ resistant to amoxicillin, ceftriaxone ceftazidime, cefotaxime, cefuroxime and aztreonam. In ICU highest resistance was seen in gentamicin 100\% followed by amikacin $85.7 \%$, netilmicin $85.7 \%$, ciprofloxacin $82.1 \%$ and imipenem $81.0 \%$ respectively. In Non ICU, antibiotic resistance was seen in gentamicin $88.9 \%$, amikacin $55.6 \%$, ciprofloxacin $54.4 \%$, netilmicin $11.1 \%$ and imipenem $22.2 \%$. Biofilm forming Acinetobacter isolates showed $7.1 \%$ colistin resistant in ICU isolates and $100.0 \%$ sensitivity in Non ICU isolates.

Resistance to most of the antibiotics is becoming common, and very few therapeutic options remain. A study from Pandicherry India showed biofilm producers of Acinetobacter isolates were $100 \%$ resistant to imipenem, amikacin $82 \%$, cephotaxime $88 \%$, ciprofloxacin $70 \%$ and aztreonam $38 \%$. ${ }^{19}$ Study in South India showed, biofilm positive Acinetobacter showed resistance to ceftazidime $95 \%$, cefepime $95 \%$, aztreonam $85 \%$, ciprofloxacin $85 \%$, amikacin $80 \%$, gentamicin $70 \%$, imipenem $65 \%$, pipercillin+tazobactum $40 \%$ and netilmicin $20 \%$. ${ }^{16}$ Another study was conducted in USA showed, 79.5\% were multi- drug resistant (MDR) $A$. baumannii. Among these, 62 were resistant to ceftazidime and 66 were resistant to imipenem. The imipenem resistant isolates were also resistant to amikacin, gentamicin, streptomycin, tetracycline, ciprofloxacin and nalidixic acid. ${ }^{20}$

\section{Conclusion}

In conclusion, the data obtained in the present work showed that most of the clinical isolates of Acinetobacter species were biofilm producers especially from device in ICU samples and they are multidrug resistant. All biofilm producing Acinetobacter species were found to be resistant to clinically achievable levels of most commonly used antibiotics such as penicillin, cephalosporin, aminoglycosides, quinolone, carbapenem and monobactam group of drugs. Polymyxins remain the only agent that may be consistently active in vitro against Acinetobacter species. However, polymixin resistant Acinetobacter isolates are slowly emerging. This is very alerming for us that biofilm forming multidrug resistant Acinetobacter species represents a severe threat in the treatment of hospitalized patients. Combination therapy may be the only therapeutic option to preserve the clinical utility of the polymixins against Acinetobacter. So, antibiotic policy and guidelines are essential to eliminate major outbreak in future.

\section{Conflict of Interest : None}

\section{References}

1. Jeong SH, Bae IK, Park KO, An YJ, Sohn SG, Jang SJ, et al. Outbreaks of imipenem- resistant Acionetobacter baumannii producing carbapenemases in Korea. J Microbiol 2006; 44: 423- 31 .

2. Josh SG, Litake GM, Satpute MG, Clinical and demographic features of infection caused by Acinetobacter species. Indian J Med Sci 2006; 60: 351-60.

3. Dijkshoorn L, Nemec A, Seifert H. An increasing threat in hospitals: Multidrug resistant Acinetobacter baumannii. Nat Rev Microbiol 2007; 5: 939- 51.

4. Prashanth K, Badrinath S. Epidemiological investigation of nosocomial Acinetobacter infections using AP- PCR and Pulse field gel electrophoresis. Indian J Med Res 2005; 122:408- 18 .

5. Jain R, Danziger LH. Multidrug- resistant Acinetobacter infections: An emerging challenge to clinicians. Ann Pharmacother 2004; 38: 1449- 59.

6. Joly-Guillou ML. Clinical Impact and Pathogenicity Of Acinetobacter. Clinical Microbiology and Infection 2005;11: 868-873.

7. Lee HW, Koh YM, Kim J, Lee JC, Lee YC, Seoll SY, Cho DT and Kim J. Capacity of multidrug-resistant clinical isolates of Acinetobacter baumannii to form biofilm and adhere to epithelial cell surfaces. Clin Microbial Infect 2008; 14:49-54.

8. Forbes BA, Sham DF, Weissfeld AS. Bailey and Scott's diagnostic Microbiology, $10^{\text {th }}$ Edition. Mosby, New York 1998: 167-87.

9. Collee JG, Miles RS, Watt B. Tests for the identification of bacteria. Mackie and McCartney Practical Medical Microbiology, EDs: Collee, Marmion, Fraser, Simmons, $14^{\text {th }}$ Edition. Churchill Livingstone, NY, 1996: 131-149.

10. Bauer AW, Kirby WMM, Sherris JC, Turek M. Antibiotic sensitivity testing by a standardized single disk method. American Journal of Clinical Pathology 1966; 45:493-496.

11. National Committee for Clinical Laboratory Standards. (2001) Performance standards for Antimicrobial Susceptibility Tests. Eleventh informational supplement. NCCLS document M100-S11 NCCLS. Wayne, Pennsylvania, USA.

12. Toledo-Arana A, Valle J, Solano C, Arrizubieta MJ, Cucarella C, Lamata M, Amorena B, Leiva J, Penades JR and Lasa I. The Enterococcal Surface Protein, ESP, Is Involved in Enterococcus faecalis Biofilm Formation. Applied and Environmental Microbiology 2001; 67(10): 4538-4545.

13. Stepanovic S, Vukovic D, Hola V, Bonaventura G D, Djukic S, Cirkovic I, Ruzicka F. Quantification of Biofilm in microtitre plates: overview for assessment of biofilm production by staphylococci. APMIS 2007; 115:891-9.

14. Costerton JW, Stewart PS, Greenberg EP. Bacterial biofilms: A common Cause of Persistent Infections. Science 1999; 284:1318-1322. 
15. Lewis K. Riddle of biofilm resistance. Antimicrob Agents Chemother 2001;45:999-1007.

16. Dheepa M, Vinitha L, Appalaraju B. Comparision of biofilm production and multiple drug resistance in clinical isolates of Acinetobacter baumannii from a tertiary care hospital in South India. Int J Pharm Biomed Sci 2011; 2(4), 103-107.

17. Rodriguez-Bano J, Marti S,Soto S, Fernandez-Cuenca F, Cisneros M, Pachon J, Pascual A, Martinez-Martinez, McQueary, Actis L, Vila J and the Spanish Group for the Study of Nosocomial Infections. Biofilm formation Acinetobacter baumannii: associated features and clinical implications. Clin Microbiol Infect 2008; 14:276-278.

18. Cevahir N, Demir M, Kaleli I, Gurbuz M, Tikvesli S. Evaluation of Biofilm production, gelatinase activity and mannose- resistant hemagglutination in Acinetobacter baumannii strains. J of Microbiology, Immunology and Infection 2008; 41:513-518.

19. Rao RS, Karthika RU, Singh SP, Shashikala P, Kanungo R, Jayachandra S, Prashanth K. Correlation Between Biofilm Production and Multiple Drug Resistance In Imipenum Resistant Clinical Isolates of Acinetobacter baumannii. Indian J of Medical Microbiology 2008; 26(4): 333-7.

20. Vijaya BS, Govindan R, Preeti P, Kurt S, Daniel T, Prapas P, Mario M, Wondwossen AG. Genetic relatedness and molecular characterization of multidrug resistant Acinetobacter baumannii isolated in central Ohio, USA. Ann Clin Microbial Antimicrob 2009, 8, 21. 